\title{
Pressure Sensor Drifts in Argo and Their Impacts
}

\author{
PAUL M. BARKER \\ Antarctic Climate and Ecosystems Cooperative Research Centre, Hobart, Tasmania, Australia \\ JEFF R. DUNN \\ Centre for Australian Weather and Climate Research, Hobart, Tasmania, Australia \\ CATia M. Domingues \\ Centre for Australian Weather and Climate Research, Aspendale, Victoria, Australia \\ SUSAN E. WIJFFELS \\ Centre for Australian Weather and Climate Research, Hobart, Tasmania, Australia
}

(Manuscript received 18 November 2010, in final form 28 February 2011)

\begin{abstract}
In recent years, autonomous profiling floats have become the prime component of the in situ ocean observing system through the implementation of the Argo program. These data are now the dominant input to estimates of the evolution of the global ocean heat content and associated thermosteric sea level rise. The Autonomous Profiling Explorer (APEX) is the dominant type of Argo float ( $\sim 62 \%)$, and a large portion of these floats report pressure measurements that are uncorrected for sensor drift, the size and source of which are described herein. The remaining Argo float types are designed to automatically self-correct for any pressure drift. Only about $57 \%$ of the APEX float profiles (or $~ 38 \%$ Argo profiles) can be corrected, but this typically has not been done by the data centers that distribute the data (as of January 2009). A pressure correction method for APEX floats is described and applied to the Argo dataset. A comparison between estimates using the corrected Argo dataset and the publically available uncorrected dataset (as of January 2009) reveals that the pressure corrections remove significant regional errors from ocean temperature, salinity, and thermosteric sea level fields. In the global mean, $43 \%$ of uncorrectable APEX float profiles (or $\sim 28 \%$ Argo profiles) appear to largely offset the effect of the correctable APEX float profiles with positive pressure drifts. While about half of the uncorrectable APEX profiles can, in principle, be recovered in the near future (after inclusion of technical information that allows for corrections), the other half have negative pressure drifts truncated to zero (resulting from firmware limitations), which do not allow for corrections. Therefore, any Argo pressure profile that cannot be corrected for biases should be excluded from global change research. This study underscores the ongoing need for careful analyses to detect and remove subtle but systematic errors in ocean observations.
\end{abstract}

\section{Introduction}

The oceans cover about $70 \%$ of the earth's surface and have a 1000-times-greater capacity to absorb heat than the atmosphere. Because the oceans store large quantities of heat, they play an important role in our

Corresponding author address: Paul Barker, CSIRO Marine and Atmospheric Research, GPO Box 1538, Hobart, Tasmania 7001 Australia.

E-mail: paul.barker@csiro.au climate and its variability. Changes in ocean heat content $(\mathrm{OHC})$ are an indicator of the planetary energy imbalance (Levitus et al. 2005; Hansen et al. 2005; Murphy et al. 2009) and are an important contribution to the global sea level budget through thermal expansion (Domingues et al. 2008). Since 1960, the oceans have absorbed about $90 \%$ of the total heat absorbed by the earth system (Bindoff et al. 2007). The thermosteric contribution dominates the global integral changes (Church et al. 2008), while halosteric effects can be important regionally. 
Because of the small but persistent size of the multidecadal signal, $\mathrm{OHC}$ estimates are very sensitive to biases in either temperature or depth measurements, especially biases that change over time. Gouretski and Koltermann (2007) detected a time-varying warm bias in expendable bathythermograph (XBT) data, which is hypothesized to be due to small manufacturing changes affecting the depth estimates of the temperature profiles (Wijffels et al. 2008; Ishii and Kimoto 2009). Corrections for this bias have provided more accurate estimates of global warming in the upper $700 \mathrm{~m}$ of the oceans for the past $50 \mathrm{yr}$ (Domingues et al. 2008; Ishii and Kimoto 2009; Levitus et al. 2009). The corrections have also allowed for more accurate estimates of thermosteric sea level rise that helped to improve the closure of the 1961-2003 sea level budget (Domingues et al. 2008), and thus resolve a key uncertainty stated in the fourth Intergovernmental Panel on Climate Change (IPCC) report (Solomon et al. 2007).

XBTs were the dominant source of ocean temperature profile data until the deployment of a global array of autonomous profiling floats by the Argo program (Roemmich et al. 1999, 2009; Gould et al. 2004; Gould 2005). Different observing technologies likely suffer different biases, and so a major shift in the dominant observing platforms can significantly impact estimates of the evolution of the global OHC. Lyman et al. (2006) reported on rapid ocean cooling that actually resulted from a combination of warm biases in XBT data and a cold bias in Argo data (Willis et al. 2007) from a particular float model [the Sounding Oceanographic Lagrangian Observer (SOLO) fitted with Falmouth Scientific Instruments (FSI) sensor package deployed by Woods Hole Oceanographic Institution (WHOI), instrument type 852]. More recently, some studies suggested that there may be a more widespread negative bias in Argo pressure measurements (Uchida et al. 2008; Uchida and Imawaki 2008).

A uniform depth error of 5 dbar globally produces a temperature bias that is greater than the observed ocean warming during the past $50 \mathrm{yr}$ in the tropical and subtropical ocean and equals almost half of the observed warming in the higher latitudes when averaging between 0 and $300 \mathrm{~m}$ (Kobayashi and Johnson 2007). Therefore, in order to have confidence in the estimates of changes in $\mathrm{OHC}$ and associated sea level changes over the 5-yr time scale of global Argo coverage to date, systematic biases in pressure must be smaller than $5 \mathrm{dbar}$. Here, we provide a description of the Argo dataset (section 2) and their pressure biases (section 3); devise a pressure bias correction method for the dominant type of Argo float (section 4); compare our corrections with corrections done by the Argo data centers (section 5); and assess the impact of the Argo pressure biases in estimates of ocean temperature (section 6), salinity (section 7), and thermosteric sea level (section 8). Section 9 provides a summary and conclusion.

\section{The Argo program and data system}

The Argo program aims to operate 3000 active freely drifting profiling floats throughout the world's ice-free open oceans (Gould et al. 2004). Since 1999, more than 5000 floats have been deployed and the array is now providing $\sim 100000$ temperature-salinity profiles per year. With lifetimes of 3-5 yr, the floats measure salinity and temperature from the surface to a depth of 2000 dbar every 10 days, drifting passively at $1000 \mathrm{dbar}$ between profiles. Argo data are available within $24 \mathrm{~h}$ of collection via the Global Data Assembly Centers (GDACs) located both in France and the United States.

The following three float types dominate the array: 1) the Autonomous Profiling Explorer (APEX), manufactured by Teledyne Webb Research (TWR; United States) comprising $\sim 62 \%$ of the deployments; 2 ) SOLO, manufactured by the Scripps Institution of Oceanography (United States) and WHOI (United States) comprising $\sim 26 \%$ of the deployments; and 3 ) the PROVOR developed by Martec MetOcean, working with Institut Français de Recherche pour l'Exploitation de la Mer (IFREMER; $\sim 12 \%$ of the floats). To date PROVOR floats have been manufactured by companies that include Tekelec, Martec, Kannad, and NKE.

Argo data are processed by national Data Assembly Centers (DACs) and converted to the Argo standard format before being transmitted to the GDACs (Carval et al. 2008) and the World Meteorological Organization Global Telecommunication System. There are two stages of quality control for Argo data: real time (R) and delayed mode (D). In the R stage, data undergo an automatic quality control screening before being sent to the GDACs, usually within $24 \mathrm{~h}$ of the profile being recorded. The D stage involves detailed scrutiny of the data by the DACs or their float provider and application of adjustments to account for instrumental drifts or other biases. On average, the higher-quality D version can take about 1-2 yr after profile acquisition to become available at the GDACs.

\section{Pressure sensors, their biases, and float controllers}

The conductivity-temperature-depth (CTD) sensors fitted to APEX floats have been manufactured by two companies-Seabird Electronics, Inc. (SBE), which is the dominant supplier for APEX floats, accounting for $99.5 \%$ of the sensors, and FSI, which supplies the 


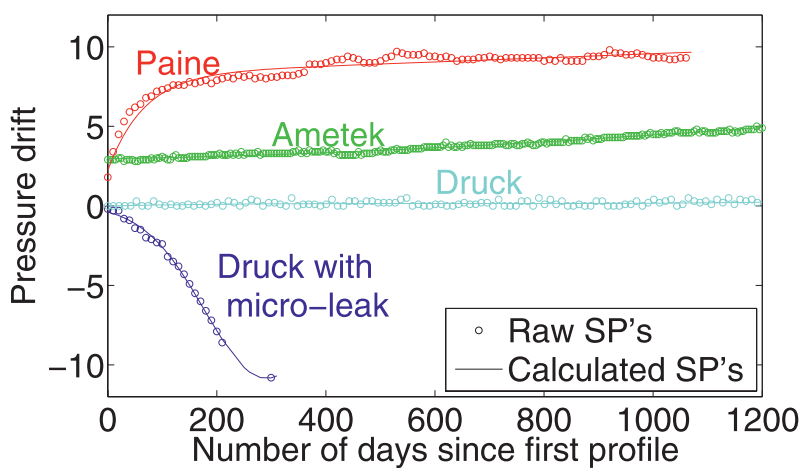

FIG. 1. Typical raw (circles) and calculated (lines) SP offsets for pressure transducers: Paine (WMO_ID 56501), Ametek (WMO_ID 2900089), Druck (WMO_ID 3900263), and Druck microleak (WMO_ID 5901649).

remaining $0.5 \%$. SBE has supplied two CTD models for use on APEX floats, the SBE-41 (which burst samples on a preset pressure table), which accounts for approximately two-thirds of the APEX CTDs, and the SBE41CP (which continuously samples), which comprises the remainder (see http://www.seabird.com/alace.htm for instrument specifications).

On APEX floats, the pressure transducer is threaded into a port on the domed housing of the float casing, adjacent to the temperature and salinity cell. The pressure sensors all work by measuring the output voltage of the sensor's strain gauge bridge, which is proportional to the applied pressure and the input voltage. These sensors are similar to the strain gauge pressure sensors that have been used on shipboard CTD instruments for decades.

When Argo started in 1999, APEX floats were fitted with pressure transducers manufactured by the Paine Corporation. However, SBE stopped using them around May 2000 because they suffered from significant sensor drift over a float deployment and exhibited a large thermal shock response. They were replaced with Ametek 3000 pressure transducers because these had both lower hysteresis and lower drift rates when aged at high pressures. Unfortunately a manufacturing defect was later discovered, which resulted in the reference chamber losing pressure. This leak caused a variable offset in the pressure measurements, manifesting as a large change in the pressure recorded at the surface (see below) over the first few profiles, which would then asymptote to a steady increasing offset for the remainder of the float life (Fig. 1). SBE once again changed their pressure sensor supplier to the Druck Corporation (a division of General Electrics) and installed Druck PDCR 1820 series pressure transducers in all of their CTDs mainly because of their stability.
Some of the early Druck models, particularly those manufactured prior to 2003, suffered from a "snowflake" problem, whereby flakes of titanium oxide spalled off from the inside of the sensor housing led to electrical arcs causing the oil in the sensor chamber to carbonize. After enough carbon tubes were generated, the cell would short and the signal would go to full scale (D. Swift 2009, personal communication). Druck has since rectified this problem and the sensors were thought to be very stable, that is, until a recent discovery of a significant number (3\% prior to 2007 and $25 \%-35 \%$ after 2007) of sensors now appear to suffer from a "microleak," whereby oil leaks from the inner sensor chamber through fine cracks in the seals at the back of the sensor (Fig. 1).

The impacts of the change in pressure sensor technology requires careful consideration because early Argo data are dominated by Paine sensors, followed by a mixture of Paine and Ametek sensors, before a slow transition to dominance by Druck sensors (Table 1). It is also important to recognize that many of the older-style sensors deployed early in Argo are still reporting at the time of writing from long-lived APEX floats ( $>5 \mathrm{yr})$.

The three dominant types of Argo floats (APEX, SOLO, and PROVOR) treat pressure sensor drifts differently. APEX floats report the raw pressure sensor output for a profile and separately measure and record the atmospheric pressure at the end of their surface satellite transmission. This surface pressure (SP) is stored in the float's memory until its next surfacing, when the SP value is transmitted with the following profile data. Thus, for APEX, SP is one transmission cycle behind the profile data. Note that all of the reported pressures are gauge pressure, which is the absolute pressure minus the atmospheric pressure. Because the gauge pressure at sea level is largely within $1 \mathrm{dbar}$ of zero, the SP reading will reflect any drift in the sensor. Thus, given a surface reading and assuming that any pressure error is not depth dependent (which is typically true based on laboratory tests and shipboard use of these sensors), the raw profile pressures can be corrected for sensor drift simply by subtracting the reported SP. SBE notes that, for the Paine and Druck pressure sensors, the primary source of error in the pressure readings comes from sensor drift, and this can be eliminated by subtracting SP from the reported profile pressures.

Most APEX floats actually transmit the surface pressure measurement with an offset of $5 \mathrm{dbar}$, which is a "stop pressure" used by the controller board to shut down the CTD pump on the next profile to avoid ingestion of the ocean surface skin layer and subsequent fouling. This also ensures that the conductivity cell remains full of clean subsurface water while the float is 
TABLE 1. Distribution of SBE-41 and SEB-41CP by pressure transducer manufacture and model.

\begin{tabular}{cccc}
\hline $\begin{array}{c}\text { Pressure sensor manufacturer } \\
\text { and model }\end{array}$ & $\begin{array}{c}\text { No. of SBE-41 } \\
\text { manufactured }\end{array}$ & $\begin{array}{c}\text { No. of SBE-41CP } \\
\text { manufactured }\end{array}$ & End of use \\
\hline Paine 1500 & 111 & 0 & Dec 1999 \\
Paine 3000 & 90 & 4 & May 2000 \\
Ametek 3000 & 575 & 320 & Aug 2002 \\
Druck & $2000+$ & $1500+$ & Still in use \\
\hline
\end{tabular}

transmitting. On APEX floats, the reporting of SP is dependent on the installed controller board, which is identified as APF $n$, where $n$ ranges between 1 and 9. By default APF5-APF8, and possible earlier series, the reported SP is restricted to values greater than zero with negative surface pressures truncated to zero. Thus, if a pressure sensor develops a negative pressure drift on floats with an APF8 or earlier series, the reported surface pressure is always zero. Here, we will refer to these floats as Truncating Negative Drift Profilers (TNDPs).

Recently TWR offered the option of a completely redesigned controller board, the APF9, developed in conjunction with the University of Washington and SBE. The APF9 is more flexible than the APF8 and has the added advantage that it reports the untruncated SP of each profile. As of January 2009, only the University of Washington and Australia have chosen to deploy APF9 APEX floats, but many other groups are now deploying these.

SOLO and PROVOR floats are currently programmed to autocorrect the recorded profile pressure on board the float. They do this by recording the SP before diving and then removing the SP from the following profile, prior to transmission. SOLO floats manufactured prior to 2002 reported the raw pressure profile and surface pressure similarly to the APEX floats. However, these data have now passed through delayed-mode quality control, where any pressure sensor drift was removed (J. Gilson 2009, personal communication).

\section{Pressure corrections for APEX floats}

Because SOLO and PROVOR floats correct for pressure drifts on board, we concentrate on correcting the raw pressure measurements from APEX floats (instrument types $831,845,846$, and 847) downloaded from the global Argo Data Assembly Centers in early January 2009. About $43 \%$ of the APEX profiles were found to be uncorrectable, and hence unsuitable for tracking global OHC (Table 2). Note that all of the SOLO floats fitted with FSI CTDs deployed by WHOI (instrument type 852), including those listed as correctable on the Argo project office Web site (http://www.argo.ucsd.edu), failed our quality control analysis of their properties (practical salinity and conservative temperature) on density surfaces, and so we also deemed them unsuitable for tracking global OHC (Table 2). The overall distribution of unusable Argo profiles per year and per rejected float case is summarized in Fig. 2.

The group of uncorrectable APEX profiles (Table 3) includes both TNDP and floats that are missing vital information that allows for their pressure correction. APEX floats were not corrected and excluded if there were insufficient SP data to 1) characterize the sensor drift (either $>60$ days shorter than the profile data record or SP reported for less than half the profiles), or 2) exhibit a truncated negative drift (assessed when $80 \%$ of the SP reported are equal to zero and the float is fitted with an APF8 or earlier series). Other floats excluded

TABLE 2. Statistics of Argo floats and profile categories.

\begin{tabular}{|c|c|c|c|c|}
\hline & $\begin{array}{l}\text { No. of floats } \\
\text { deployed }\end{array}$ & $\begin{array}{c}\text { Percent of total } \\
\text { Argo floats }\end{array}$ & $\begin{array}{l}\text { No. of } \\
\text { profiles }\end{array}$ & $\begin{array}{l}\text { Percent of total } \\
\text { Argo profiles }\end{array}$ \\
\hline Correctable APEX floats & 1673 & 29.4 & 187868 & 37.7 \\
\hline $\begin{array}{l}\text { Non-APEX floats (SOLO except FSI deployed by } \\
\text { WHOI, PROVOR, Nemo, Ninja) }\end{array}$ & 1865 & 33.0 & 139530 & 27.9 \\
\hline Total usable Argo floats & 3538 & 62.4 & 327398 & 65.6 \\
\hline Uncorrectable APEX floats (individual cases listed in Table 3) & 1849 & 32.6 & 140303 & 28.2 \\
\hline $\begin{array}{l}\text { All SOLO FSI deployed by WHOI (discarded: failed our } \\
\text { quality control) }\end{array}$ & 279 & 4.9 & 30961 & 6.2 \\
\hline Unidentifiable floats & 7 & 0.1 & 59 & 0.01 \\
\hline Total unusable Argo floats & 2135 & 37.6 & 171323 & 34.4 \\
\hline Argo floats listed on GDAC & 5673 & 100 & 498721 & 100 \\
\hline
\end{tabular}




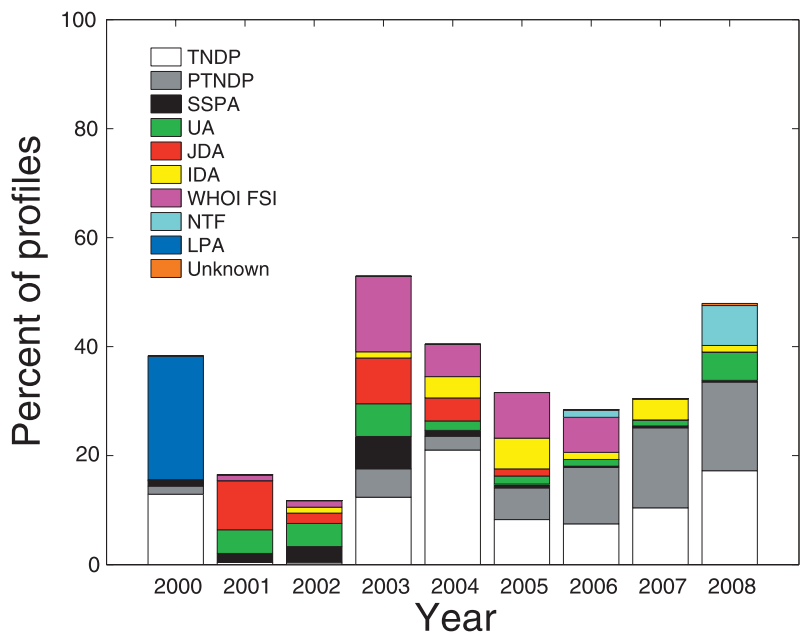

FIG. 2. Percentage and makeup of total number of unusable profiles (Table 2) collected by deployment year in the Argo database as of Jan 2009: TNDP; Possible Truncating Negative Drift Profilers (PTNDPs), where all surface pressures are truncated but the SP record is too short to confirm this; Short Surface Pressure APEX (SSPA) floats, where the SP record is too short to fit a correction; unusable APEX (UA) for less than four surface pressure readings, usually resulting from recent deployment; JAMSTEC/ JMA-deployed APEX (JDA), with missing raw pressures; Indiandeployed APEX (IDA); WHOI FSI are SOLO floats equipped with FSI pressure transducers deployed by WHOI; floats with no technical file (NTF); low-precision floats APEX (LPA); and unknown types of floats.

are all APEX floats deployed by India, resulting from a fault in their reported SPs, and approximately 15000 profiles collected by the Japan Marine Science and Technology Center (JAMSTEC) and the Japan Meteorological Agency (JMA) in which the raw pressure data were overwritten by incorrectly adjusted values. We have also opted to exclude 24 of the early model APEX floats because the precision of the reported SP is 5 dbar and, therefore, it is too low for calculating global OHC (and thermosteric sea level) estimates. Overall, about half of the uncorrectable APEX profiles (Table 3) are due to controller board truncation of the SP while the other half are due to incomplete processing of the technical data stream by the DACs, which, in theory, makes them recoverable in the future.

An APEX float's SP record can often be noisy and incomplete, which is not surprising because it is a single snapshot of the atmospheric pressure recorded by the float prior to submerging, and it is significantly affected by waves and satellite transmission errors. Most APEX floats operate on a preprogrammed cycle and do not have the ability to store the profile history; if a float fails to surface, then that profile will be lost and the recorded surface pressure will be the shallowest pressure the float reached on that profile. Substantial noise could thus be introduced by naively interpolating between raw surface pressure values. To minimize the effect of these errors in the surface pressure record and allow for sensible interpolation across gaps and some extrapolation, a parametric curve was fitted to the raw SP reports.

The SP data were initially despiked to remove obvious bad data points (typically differences greater than 60 dbar) occurring when the previous profile did not reach the ocean surface (e.g., when a float stuck on the mud on the bottom of the ocean) or there was a transmission error. This was then followed by finer despiking, where differences in consecutive measurements were not allowed to be greater than 5 dbar for APF8s or earlier series and $9 \mathrm{dbar}$ for APF9s, although it was found that it was necessary to increase the pressure change limit for APEX floats released before 2000 when the fast-drifting Paine sensors were being used.

There were smaller, subtler spikes that continued to influence the time series (Fig. 1). Thus, to achieve a better estimate of the pressure sensor drift, the data were smoothed with a linear least squares fitting and a firstdegree polynomial using robust fitting spanning $20 \%$ of the data series (lowess; Cleveland 1979, 1981). This removed the effect of short-period fluctuations and had the added benefit of being insensitive to the remaining

TABLE 3. Statistics of uncorrectable APEX floats and profile categories.

\begin{tabular}{|c|c|c|c|c|}
\hline & $\begin{array}{l}\text { No. of floats } \\
\text { deployed }\end{array}$ & $\begin{array}{l}\text { Percent of total } \\
\text { Argo floats }\end{array}$ & $\begin{array}{l}\text { No. of } \\
\text { profiles }\end{array}$ & $\begin{array}{l}\text { Percent of total } \\
\text { Argo profiles }\end{array}$ \\
\hline Truncated negative drifting APEX (negative values truncated to zero) & 612 & 10.8 & 53823 & 10.8 \\
\hline Possible negative drifting APEX ( $>60$ days of SP missing) & 406 & 7.2 & 29384 & 5.9 \\
\hline Short Surface Pressure APEX ( $>60$ days of SP missing) & 84 & 1.5 & 7868 & 1.6 \\
\hline $\begin{array}{l}\text { Unusable APEX (typically recently released or surface pressures } \\
\text { are missing after despiking) }\end{array}$ & 272 & 4.8 & 13126 & 2.6 \\
\hline No technical file & 34 & 0.6 & 2647 & 0.5 \\
\hline Indian-deployed APEX (invalid surface pressures) & 132 & 2.3 & 14545 & 2.9 \\
\hline Japanese-deployed APEX (missing raw pressures) & 285 & 5 & 15296 & 3.1 \\
\hline Low precision APEX & 24 & 0.24 & 3614 & 0.7 \\
\hline Total uncorrectable APEX floats & 1849 & $\sim 33$ & 140303 & $\sim 28$ \\
\hline
\end{tabular}



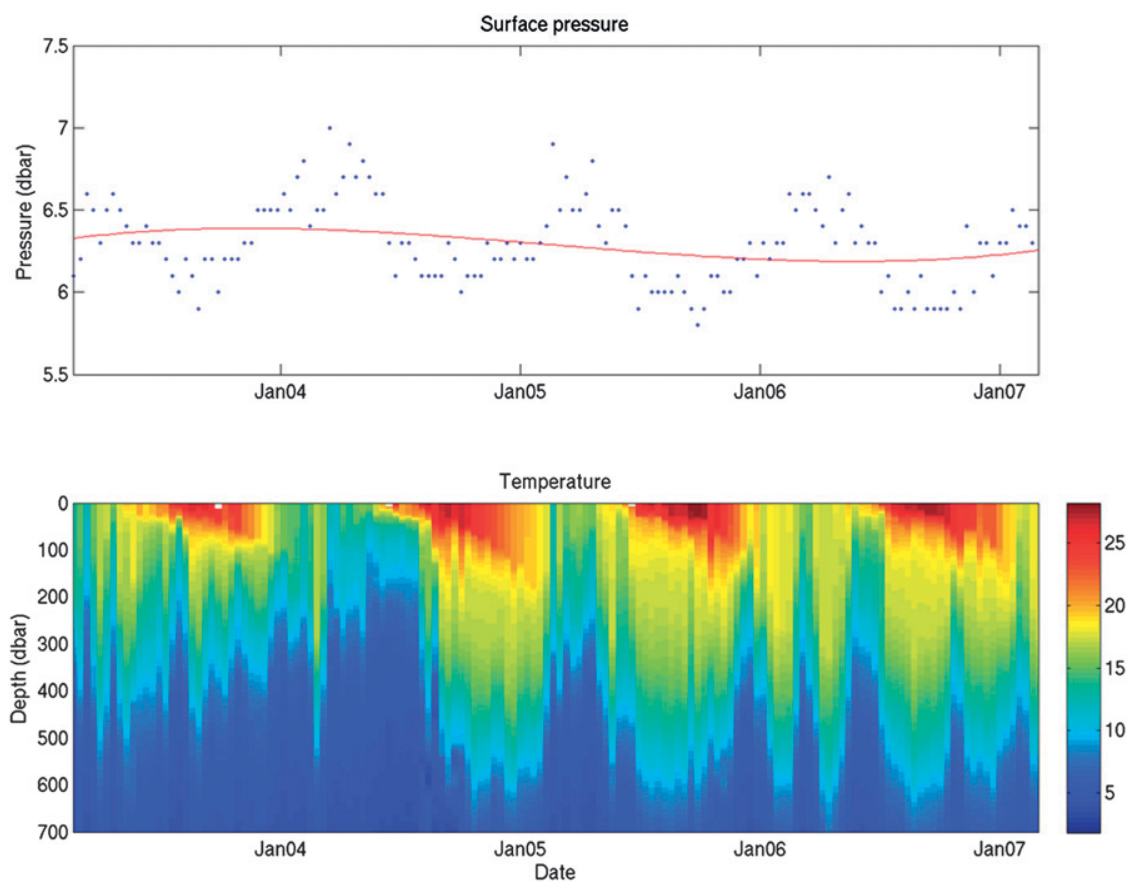

FIG. 3. (top) Surface pressure (dbar) time series for APEX float WMO_ID 2900253: raw (blue) and calculated (red). (bottom) Corresponding temperature $\left({ }^{\circ} \mathrm{C}\right)$ time series over depth (surface-700 dbar).

outliers. This particular method was adopted because it is able to handle irregular time steps and the span of the weights was set to be a percentage of the length of the time series.

A time-varying function was fitted to the resulting heavily smoothed SP time series. It was not possible to apply a single mathematical form to all of the cases because the behavior of the drifts was highly variable across the sensor types (Fig. 1). Ten parametric models were tested for each float, and the model that had the lowest sum of the squared residuals was selected. It was important that the independent variable was time (and not profile number) dependent because some floats do not cycle at regular intervals. This is especially true for the APF9 floats. Across the entire Argo dataset, we found that we could confidently correct $57 \%$ of the APEX profiles (Table 2), which in turn corresponds to $38 \%$ of the total number of Argo profiles.

An interesting phenomenon that became evident is the impact of the near-surface temperature gradients in the seasonal thermocline on pressure sensor biases in the subtropics, typically seen in Druck pressure transducers. After the float passes through the strong summer seasonal thermocline the reported surface pressure reading is about $1 \mathrm{dbar}$ lower than in winter when the thermocline is eroded (Fig. 3). We believe this is a thermal lag error in the pressure sensor. Ideally, corrections for this error should only be applied to the water column above the thermocline. We have not attempted to adjust the recorded pressures for this effect (usually within 2.4 dbar, the specified accuracy of the sensor), because our smoothing method averages through the seasonal cycle to resolve the lower-frequency drift (Fig. 3, top panel).

With the exception of Druck sensors with microleaks, the largest pressure corrections are deduced for floats deployed prior to 2004 (Fig. 4), when the majority of these float were fitted with either Paine or Ametek transducers. The year 2003 was a transition year for SBE CTDs when the Druck transducers were deployed, resulting in a significant decrease in the magnitude of the SP biases (Fig. 4). Also, during 2003, intermittent cases occur of floats requiring large corrections where programs deployed their older stock of APEX floats fitted with Paine or Ametek transducers. The number of floats deployed in this transition period was lower than that initially anticipated because of the Druck snowflake problem, which halted global Argo deployments for nearly $1 \mathrm{yr}$, as evidenced by the low concentration of corrections during 2003 (Fig. 4). Since 2004, the majority of the pressure sensor drifts were closer to manufacturer's accuracy of 2.4 dbar.

\section{Comparison with DAC pressure corrections}

Although we correct for positive and negative pressure biases in APEX floats (Fig. 4), most of the corrections 


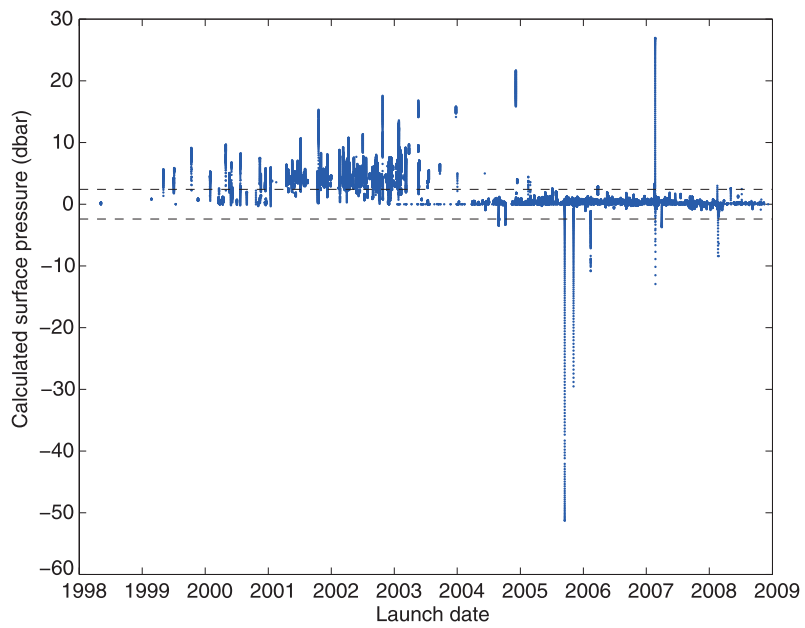

FIG. 4. Our calculated surface pressure drifts for all correctable APEX floats vs launch date. Manufacturer's accuracy of $2.4 \mathrm{dbar}$ is indicated (black dashed line). Most of the corrections are for positive surface pressure drifts.

are for positive biases (with the pressure sensor reading high of true). This reflects the fact that we had to discard APEX floats with APF8 and earlier model controller boards because their negative biases are truncated to zero (resulting from firmware limitations) and do not allow for corrections (TNDP in Table 3).

The majority of the pressure corrections that the DACs have applied to APEX profiles have been in the North Pacific and eastern Indian Ocean (Fig. 5, top panel); very few corrections were applied to APEX data outside of these two regions. The distribution of our pressure corrections (Fig. 5, middle panel) is significantly different than that of the DACs. We have identified that corrections are required throughout all of the oceanic basins (Fig. 5, bottom panel), but the largest concentration and magnitudes of corrections are in the North Atlantic, North Pacific, and Indian Oceans. The regional distribution is not unexpected because these are the regions where Argo deployments began (thus, using APEX floats fitted with Paine and Ametek transducers), with the other areas seeded with floats only relatively recently. An example of the more stable Druck transducers is the South Pacific Ocean, which has relatively few pressure corrections. The South Atlantic also has very few corrections because most of the floats were SOLO fitted with FSI CTDs, deployed by WHOI, and were excluded from our study (as explained in section 4).

The APEX pressure biases are both latitude and time dependent (Fig. 6), with the largest corrections required for data collected prior to 2005 (the Paine and Ametek era). Post-2005, the average bias is well within the Argo estimate of pressure sensor accuracy of $2.4 \mathrm{dbar}$, which reflects the excellent stability of most of the Druck
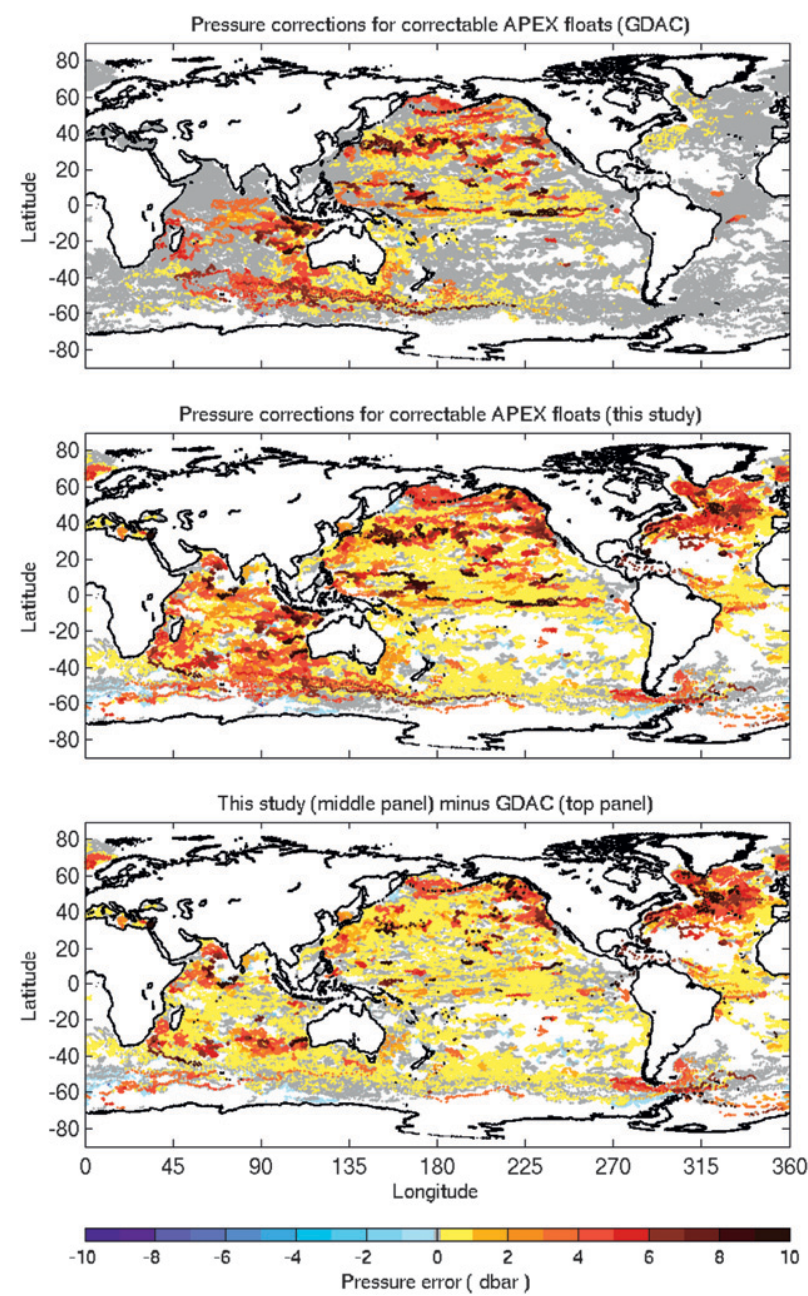

FIG. 5. Regional distribution of correctable APEX profiles colored by the amplitude of associated pressure corrections (dbar). (top) Pressure corrections applied to correctable APEX floats in the GDAC Argo dataset as of Jan 2009. (middle) Pressure corrections applied to correctable APEX floats in this study. (bottom) Pressure biases in correctable APEX floats in the GDAC dataset (this study minus GDAC version).

transducers. The large negative bias from 2007 to 2008, in the Northern Hemisphere, is due to the University of Washington APF9 floats, which do not truncate negative surface pressures.

\section{Temperature errors}

The in situ temperature errors associated with pressure biases in correctable APEX floats (Fig. 5, middle panel; Table 2) vary geographically (Fig. 7), in depth (Fig. 8), and in time (Fig. 9). Positive errors indicate that ocean temperatures have been overestimated (warmer than true). 


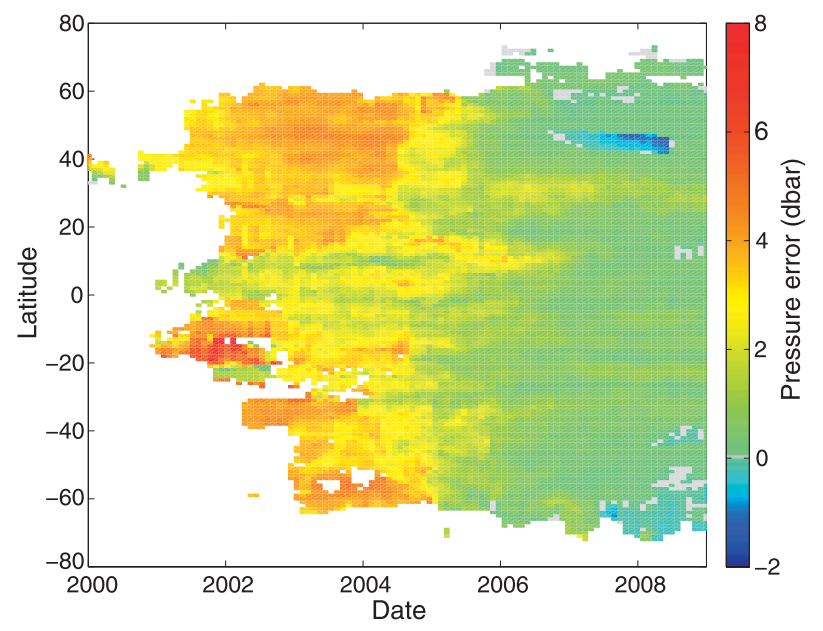

FIG. 6. Monthly $5^{\circ}$ latitude averages of pressure biases in correctable APEX floats in the GDAC dataset (this study minus GDAC version).

The regional distribution of the temperature errors in correctable APEX floats (Fig. 7), vertically averaged between 20 and $700 \mathrm{dbar}$, shows that they tend to be slightly positive in the Southern Ocean, just north of the path of the Antarctic Circumpolar Current $\left(\sim 40^{\circ} \mathrm{S}\right)$. In the Atlantic Ocean, a cluster of large positive errors is found along the path of the western boundary currents off of the east coast of North America $\left(\sim 20^{\circ}-60^{\circ} \mathrm{N}\right)$. The equatorial Atlantic $\left(\sim 20^{\circ} \mathrm{N}-20^{\circ} \mathrm{S}\right)$ also displays a cluster of positive errors while the South Atlantic is mostly devoid of APEX floats. Negative errors are mostly confined to the northeastern coast of the United States. In the Indian Ocean, large positive errors occur in the Arabian Sea $\left(\sim 20^{\circ} \mathrm{N}\right)$ and extend in a band from the east coast of South Africa $\left(\sim 30^{\circ} \mathrm{S}\right)$ to the west Australian coast. The eastern sections of the equatorial and south Indian Ocean, along the west Australian coast, have a cluster of negative errors. In the Pacific Ocean, there is a mixture of positive and negative errors spread over the equatorial and North Pacific Ocean. A cluster of large positive errors is found along the west coast of North America $\left(\sim 60^{\circ}-20^{\circ} \mathrm{N}\right)$ and in the western Pacific adjacent to the Asian coast. Some large negative errors occur in the northeast Pacific while the South Pacific is devoid of APEX floats.

Approximately $3 \%$ of the correctable APEX profiles are free from vertically averaged in situ temperature errors, $23 \%$ contain negative errors, and the vast majority (74\%) has positive errors, resulting in overestimated temperatures. These positive errors are, on average, 2-3 times greater than the negative errors. In the global mean, the positive errors dominate at all depths (Fig. 8 ), with the largest values in the upper $200 \mathrm{~m}$ and maximum $\left(-0.02^{\circ} \mathrm{C}\right)$ matching the steepest gradient of the shallow thermocline.

The zonally and vertically averaged time series of correctable APEX floats (Fig. 9) shows that the largest positive temperature errors occur prior to 2005, but persist with smaller amplitudes in the following years. One notable feature that occurs in the mid- to high latitudes is the seasonal signal peaking during the Northern Hemisphere summer months (June-August) associated with the annual development of the seasonal thermocline. A corresponding signal, offset by 6 months, although with smaller amplitude, can just be identified at similar latitudes in the Southern Hemisphere.

Given that we cannot develop corrections for APF8 or earlier APEX float series, with negative drifting pressure sensors where SP data are truncated to zero (TNDP floats, Table 3), we grossly estimate their median bias by comparing the TNDP profiles with the closest good profile (a buddy with 3-months time and $175-\mathrm{km}$ distance). Averaged across all TNDP profiles with buddies, we find a depth-independent pressure error of about -3 dbar. This median error can introduce a significant global mean cold temperature bias; however, TNDP floats are not spread uniformly throughout the oceans, with the highest concentrations in the western Pacific and Indian Oceans. Thus, we recommend that all of the TNDP profiles (about $17 \%$ of the total number of Argo profiles) be removed from studies of $\mathrm{OHC}$ and decadal changes.

\section{Salinity errors}

The practical salinity errors we report here are only due to the pressure biases in correctable APEX floats (Fig. 5, middle panel; Table 2). That is, vertical shifts of the pressure profiles affect the depth of the practical salinity observations. Note that positive errors indicate that the ocean salinities have been overestimated.

The regional distribution of the practical salinity errors in correctable APEX floats (Fig. 10), vertically averaged for 20-700 dbar, is similar to, but smaller than, the salinity change between Argo and World Ocean Atlas 2001 (WOA01) (Roemmich and Gilson 2009); and nearly everywhere the value is less than the Argo target for practical salinity accuracy of 0.01 . The uncorrected Argo data tend to underestimate the salt content in areas of the North Pacific Ocean (with largest errors off the western coast of North America), in the South Pacific Ocean closer to Australia, in the eastern Indian Ocean, in the South Atlantic Ocean near the Antarctica Peninsula, and along the western boundary current paths in the North Atlantic Ocean. The positive salinity errors lead to an overestimation of the salt content in the western and South Indian Ocean and in widespread 

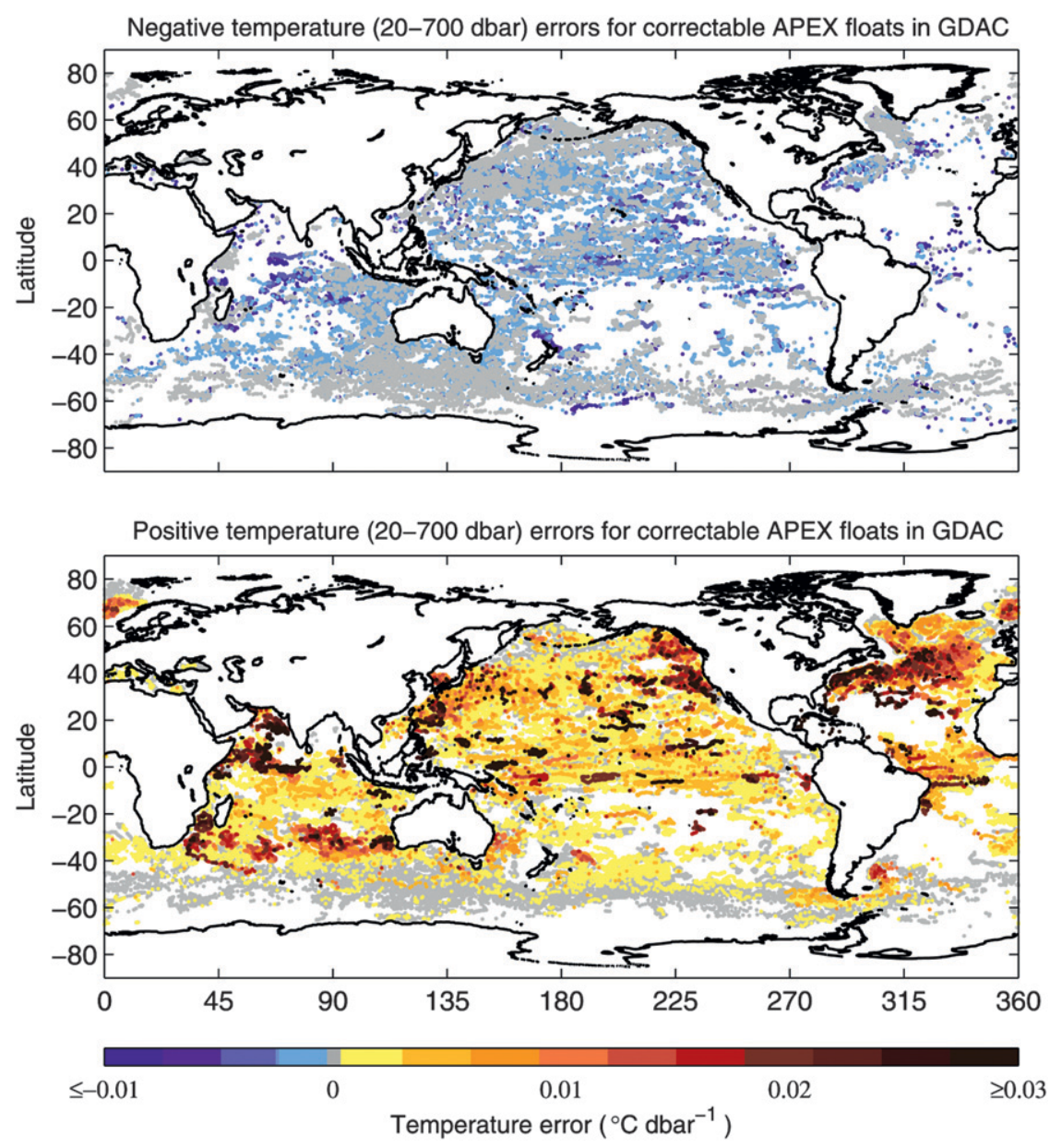

FIG. 7. Regional distribution of in situ temperature errors, vertically averaged from 20 to 700 dbar, resulting from biases in correctable APEX float profiles in the GDAC dataset: (top) negative and (bottom) positive errors. The temperature error is defined as $(1 / \Delta p)\left(\int_{p 1}^{p 2} T_{\mathrm{GDAC}} d p-\right.$ $\left.\int_{p 1}^{p 2} T_{\text {Corrected }} d p\right)$, where $T_{\mathrm{GDAC}}$ is the uncorrected dataset and $T_{\text {Corrected }}$ is our pressure-corrected dataset, and p1 and p2 are 20 and 700 dbar, respectively. Positive in situ temperature errors indicate that ocean temperatures have been overestimated (i.e., warmer than true).

areas of the Pacific Ocean (off the Asian coast and tropics), and they are clustered in the subtropical gyre of the North Atlantic Ocean and off the northeast coast of South America. We note that the distribution of the positive-negative salinity errors is generally equivalent to the temperature error distribution in Fig. 7.

\section{Thermosteric sea level errors}

To assess the impact of Argo pressure biases in thermosteric sea level from 2000 to 2008, we constructed three Argo dataset versions: 1) the overall GDAC Argo dataset, as downloaded from the Web site in January 2009 , using D data when available; 2) our version of the Argo dataset (pressure corrected), including only the APEX floats we could correct for pressure biases, and all usable SOLO and PROVOR floats (Table 2).
Further, we only kept the vertical profiles that had both temperature and salinity observations and passed our climatology-based quality control; and 3) the same as in 2 above but without applying corrections for APEX pressure biases, which we refer as pressure correctable. Most of the profiles that failed our quality control for 2 and 3 were all of the WHOI-deployed SOLO FSI floats (Table 2), including those listed as correctable on the Argo program Web site (http://www.argo.ucsd.edu).

In effect, the pressure-corrected dataset provides the best unbiased estimates of thermosteric sea level. The pressure-correctable version allows us to reveal the dominant global-scale impact of positive pressure biases in correctable APEX floats (negative pressure biases in correctable APEX floats exist, but have a minor impact). The GDAC version is the closest dataset to what 


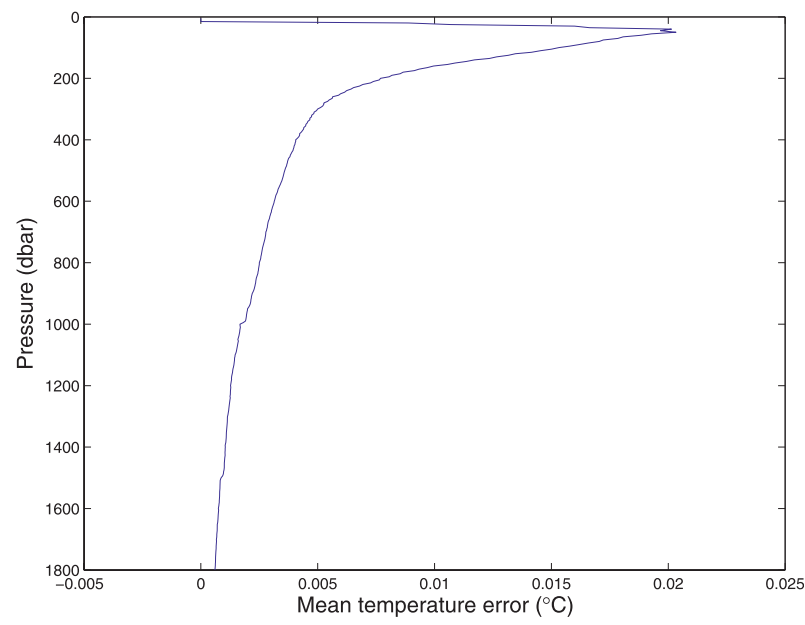

FIG. 8. Global mean in situ temperature errors over depth in the correctable APEX cohort of the GDAC Argo dataset. The in situ temperature error is defined as the GDAC minus our pressurecorrected version.

Argo users would generally employ for their own calculations, and it allows us to show the combined impact of correctable and uncorrectable APEX pressure biases, and to a smaller degree the impact of other profiles that failed our quality control (e.g., WHOI SOLO FSI floats). In terms of total number of Argo (APEX) profiles, the correctable APEX floats make up about $38 \%$ $(57 \%)$ of the profiles, the uncorrectable APEX floats about $28 \%(43 \%)$, and the WHOI SOLO FSI floats about $6 \%$. Note that the uncorrectable cohort in the GDAC version contains all of the APEX profile categories listed in Table 3, including the TNPD floats with

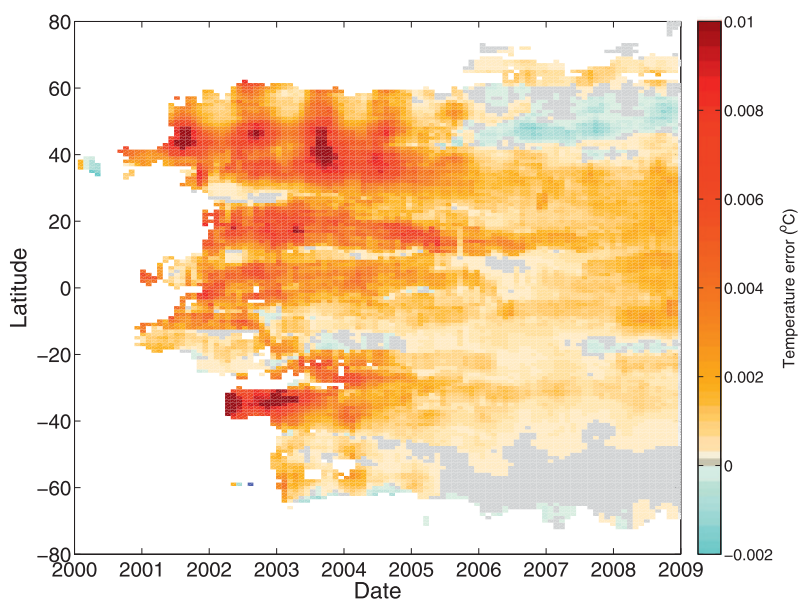

FIG. 9. Monthly $5^{\circ}$ latitude averages of vertically averaged in situ temperature errors (from 20 to 700 dbar) resulting from biases in correctable APEX float profiles in the GDAC dataset. The in situ temperature error is defined as the GDAC minus our pressurecorrected version.

negative pressure biases truncated to zero resulting from firmware limitations.

Because there are not enough data in the early years of the Argo program to estimate thermosteric sea level at a global scale, we have merged the temperature observations from bottles, CTDs, and bias-corrected XBTs (Wijffels et al. 2008) in the Enhanced Ocean Data Assimilation and Climate Prediction (ENACT)ENSEMBLES version 3 (EN3) dataset (Ingleby and Huddleston 2007). The overlapping period between the EN3 and Argo datasets is from 2000 to 2004. Thus, from 2005 onward, there are only Argo data.

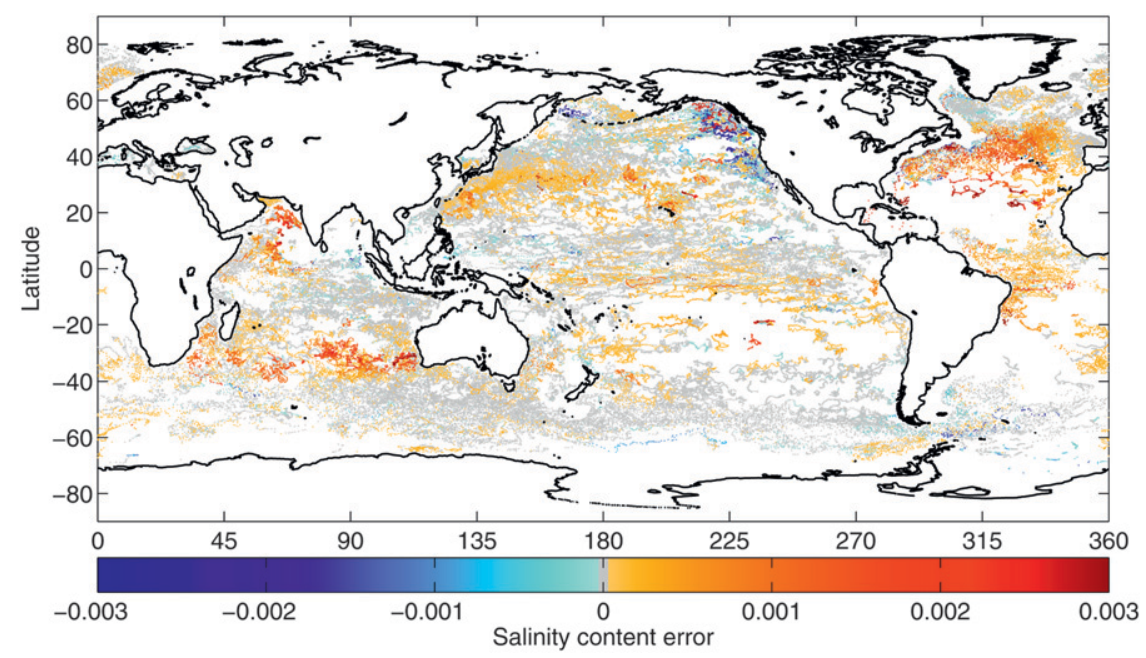

FIG. 10. Regional distribution of practical salinity errors, vertically averaged from 20 to 700 dbar, resulting from biases in correctable APEX float profiles in the GDAC dataset. The practical salinity error is defined as the GDAC minus our pressure-corrected version. 
The temperature observations were converted into monthly thermosteric sea level anomalies, from the surface to $700 \mathrm{~m}$ in incremental steps of $50 \mathrm{~m}$. Anomalies were formed by taking out a monthly mean climatology, which only used bottle, CTD, and pressure-corrected Argo data, as described in Wijffels et al. (2008). We then used a reduced-space optimal interpolation technique (Kaplan et al. 2000), as in Domingues et al. (2008), to construct spatially complete fields.

The impact of the pressure biases in the GDAC and pressure-correctable datasets for the 0-700-m depthintegrated estimates of global mean thermosteric sea level (Fig. 11, top panel) lie within the one standard deviation error bars of the pressure-corrected time series, our best unbiased estimate. The monthly GDAC time series (Fig. 11, middle panel) contain mixed biases with globally averaged compensating errors over the upper $700 \mathrm{~m}$. The uncorrectable APEX float profiles (Tables 2 and 3), including negative pressure biases, appear to largely offset, in most years, the dominant global-scale effect of the positive pressure biases in the $57 \%$ of correctable APEX floats (Fig. 11, bottom panel). The latter tends to be stronger in the upper $200-300 \mathrm{~m}$.

The largest depth-integrated errors for the pressurecorrectable global mean thermosteric sea level time series (Fig. 11, top panel) are found between 2003 and 2005 , and tend to decay toward more recent years. These errors imply a smaller global upper-ocean thermosteric sea level rise from 2003 to 2008 (although within the error bars of the pressure-corrected time series), and mostly reflect the temperature problems shown in Figs. 7-9. The large temperature errors evident in Fig. 9 during 2001/02 are not apparent in thermosteric sea level because the number of data in EN3 dominates the overall dataset in the first few years (2000-02). Also, we note that the difference between the GDAC and pressure-correctable time series in 2008 (Fig. 11, top panel) mainly arises due to some bad GDAC data passing the real-time automatic quality control. Thus, it is recommended that the higherquality data (D), which take a longer time to become available in the GDACs, be used for these sensitive calculations. At the time we performed the download, only $20 \%$ of the data for 2008 were in D.

Significant regional biases in 0-700-m depth-integrated thermosteric sea level, from 2003 to 2007, are apparent in both the mean and variance of the differences between the GDAC and pressure-corrected estimates (Fig. 12). Excluding the regional seas, which are very difficult to accurately resolve, the central-north Pacific, westernequatorial and South Pacific, and subtropical North Atlantic all feature an underestimated thermosteric sea level, while the rest of the world mostly shows an overestimation. Thus, despite the near cancellation of the
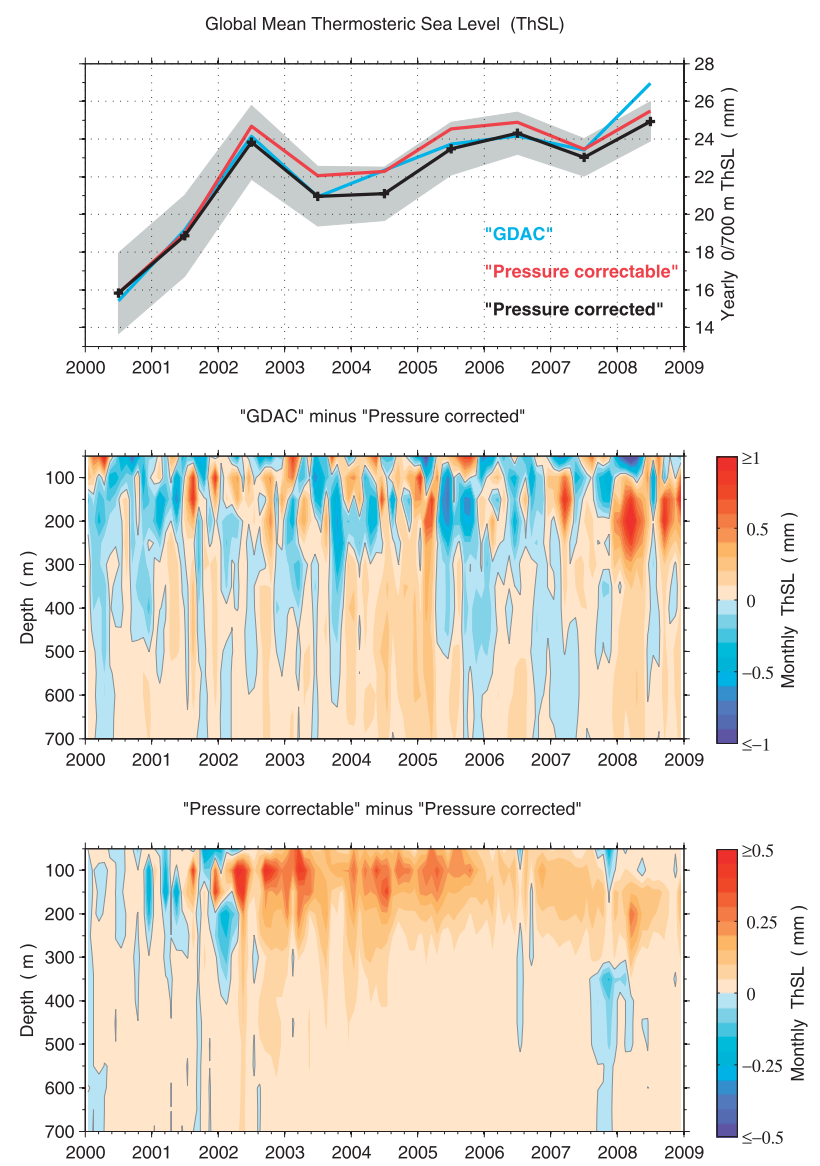

FIG. 11. Global mean thermosteric sea level time series ( $\mathrm{mm}$ ). (top) The surface-700-m depth-integrated yearly estimates for GDAC (blue), pressure correctable (red), and pressure corrected (black). One standard deviation error bars for pressure corrected are shown (gray shading). (middle) Difference between monthly GDAC and pressure corrected for the upper $700 \mathrm{~m}$. (bottom) Same as middle, but for pressure correctable and pressure corrected.

impact of the GDAC pressure errors in the global average (Fig. 11, middle panel), regional patterns appear more sensitive to these biases.

\section{Summary and conclusions}

Subtle instrumental errors can limit our ability to identify climate and sea level change signals because of the very low signal-to-noise ratio of these calculations.

In recent years, autonomous profiling Argo floats have become the prime component of the in situ ocean observing system. APEX is the dominant Argo float type, and a large portion of their pressure measurements are uncorrected for sensor drift (as of January 2009). The remaining Argo float types are designed to automatically self-correct for any pressure drift.

We developed and applied a multistep technique to identify and correct pressure biases in APEX floats. 

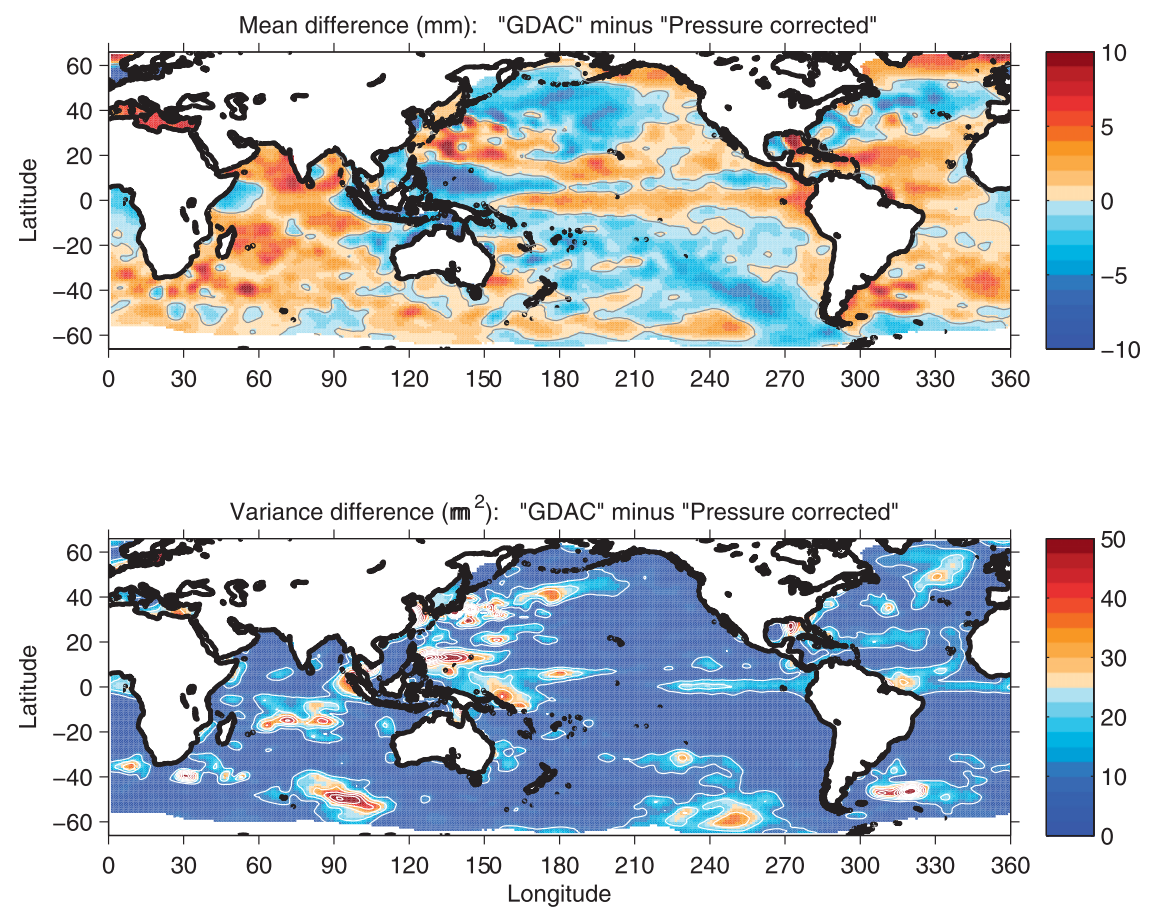

FIG. 12. Regional differences in thermosteric sea level between GDAC and pressure-corrected datasets over 2003-07. (top) Mean error (mm); positive errors indicate thermosteric sea level higher than true, and vice versa. (bottom) Variance error $\left(\mathrm{mm}^{2}\right)$.

The technique takes into account the widely variable nature of the pressure drifts and assumes that their amplitude is constant over depth. The time series of reported surface pressure values from each float is used to predict the corrections for all profiles using an optimally fitted parametric model. This calculation should be routinely executed as each float series is extended.

In early 2009, $62 \%$ of the Argo floats were APEX, and approximately $57 \%$ of their profile data could be confidently corrected for pressure biases. These systematic errors lie mostly within 5 dbar but can be as large as 20 dbar. The majority of the APEX floats, which required the greatest pressure corrections (Paine and Ametek transducers), were released prior to 2004, but many are still actively reporting data (at the time of writing). Although positive and negative pressure biases were corrected, the bulk of the corrections were done for positive biases. About $43 \%$ of the APEX profiles are uncorrectable-half of this number due to firmware limitations, where negative pressure drifts are truncated to zero. Other reasons for uncorrectable APEX floats are insufficient surface pressure data, unknown float type, and missing technical files. A comprehensive list of these "uncorrectable" floats (which thus should be discarded for global change research) has been compiled (see http://www.marine.csiro.au/ cow074/quota/argo_ offsets.htm). When updated metafiles and technical files are released we expect the percentage of APEX pressure-corrected profiles to increase by more than $10 \%$. Steps are being taken by the Argo national programs to deliver pressure-corrected APEX floats via the GDACs (http://www.argo.ucsd.edu/Acpres_drift_apex.html).

The net effect of the positive APEX pressure biases is to overestimate the temperature in the oceans and to create errors of about $10 \%$ of the magnitude of salinity differences between Argo and WOAO1 datasets. The largest temperature errors occur in the upper $200 \mathrm{~m}$ of the water column (due to the steep thermocline gradient), prior to 2005. This, in turn, incorrectly implies a smaller global mean upper-ocean warming and thermosteric sea level rise from 2003 to 2008.

In our $0-700-\mathrm{m}$ global mean thermosteric sea level estimates using data from all platforms, Argo pressure errors are imperceptible before 2002 because of the comparatively low number of Argo profiles. After 2002, negative biases from uncorrectable (and other unusable) profiles appear to compensate positive biases from correctable APEX profiles, in the global mean. Without correcting APEX pressure biases, our 0-700-m global mean thermosteric sea level estimates still lie within the one standard deviation error bars of our best unbiased estimate (corrected for APEX pressure biases and excluding all unusable Argo profiles). This suggests that the Argo pressure errors are too small to be detected 
in independent estimates of global (thermo)steric sea level inferred from altimetry and GRACE, and thus should not affect the closure of the sea level budget over 2003(/04)-08 (Cazenave et al. 2009; Leuliette and Miller 2009). Although there is near compensation in the global average, the regional thermosteric sea level patterns appear to be more sensitive to the Argo pressure biases.

This paper underscores the continuous need for careful analyses to detect and remove subtle errors in ocean observations, and also the need for complete and accurate technical data and metadata. While intercomparisons with satellite observations, such as altimeter and GRACE, are useful (Willis et al. 2008; Cazenave et al. 2009; Guinehut et al. 2009), these type of analyses alone are not sufficient. Recent studies have highlighted the importance of high-quality shipboard CTD data in the detection and correction of subtle biases in historical data collected by expendable (XBT) and mechanical (MBT) bathythermographs (Gouretski and Koltermann 2007; Wijffels et al. 2008; Ishii and Kimoto 2009; Levitus et al. 2009). This type of intercomparison would be similarly important to enhance the calibration of Argo floats.

Acknowledgments. This work has been undertaken as part of the Australian Climate Change Science Program, funded jointly by the Department of Climate Change, the Bureau of Meteorology, and CSIRO. CMD was supported by a CSIRO Office of the Chief Executive (OCE) Postdoctoral Fellowship. We thank John Church for his guidance and support, and Ann Thresher and Neil White for reviewing early drafts of this manuscript. We also thank two anonymous reviewers whose suggestions helped to improve this manuscript.

\section{REFERENCES}

Bindoff, N. L., and Coauthors, 2007: Observations: Oceanic climate change and sea level. Climate Change 2007: The Physical Science Basis, S. Solomon et al., Eds., Cambridge University Press, 385-432.

Carval, T., and Coauthors, 2008: Argo data management user's manual, version $2.1,60 \mathrm{pp}$.

Cazenave, A., K. Dominh, S. Guinehut, E. Berthier, W. Llovel, G. Ramillien, M. Ablain, and G. Larnicol, 2009: Sea level budget over 2003-2008: A reevaluation from GRACE space gravimetry, satellite altimetry and Argo. Global Planet. Change, 65, 83-88.

Church, J. A., N. J. White, T. Aarup, W. S. Wilson, P. L. Woodworth, C. M. Domingues, J. R. Hunter, and K. Lambeck, 2008: Understanding global sea levels: Past, present and future. Sustainability Sci., 3, 9-22, doi:10.1007/s11625-008-0042-4.

Cleveland, W. S., 1979: Robust locally-weighted regression and smoothing scatterplots. J. Amer. Stat. Assoc., 74, 829-836.
_ 1981: LOWESS: A program for smoothing scatterplots by robust locally-weighted regression. Amer. Stat., 35, 54.

Domingues, C. M., J. A. Church, N. J. White, P. J. Gleckler, S. E. Wijffels, P. M. Barker, and J. R. Dunn, 2008: Improved estimates of upper-ocean warming and multi-decadal sea level rise. Nature, 453, 1090-1094, doi:10.1038/nature07080.

Gould, J., 2005: From swallow floats to Argo-The development of neutrally buoyant floats. Deep-Sea Res. II, 52, 529543.

—_ and Coauthors, 2004: Argo profiling floats bring new era of in situ ocean observations. Eos, Trans. Amer. Geophys. Union, 85, 185, doi:10.1029/2004EO190002.

Gouretski, V., and K. P. Koltermann, 2007: How much is the ocean really warming? Geophys. Res. Lett., 34, L01610, doi:10.1029/ 2006 GL027834.

Guinehut, S., C. Coatanoan, A. L. Dhomps, P. Y. Le Traon, and G. Larnicol, 2009: On the use of satellite altimeter data in Argo quality control. J. Atmos. Oceanic Technol., 26, 395-402.

Hansen, J., and Coauthors, 2005: Earth's energy imbalance: Confirmation and implications. Science, 308, 1431-1435, doi:10.1126/ science. 1110252.

Ingleby, B., and M. Huddleston, 2007: Quality control of ocean temperature and salinity profiles-Historical and real-time data. J. Mar. Syst., 65, 158-175.

Ishii, M., and M. Kimoto, 2009: Reevaluation of historical ocean heat content variations with time-varying XBT and MBT depth bias corrections. J. Oceanogr., 65, 287-299.

Kaplan, A., Y. Kushnir, and M. A. Cane, 2000: Reduced space optimal interpolation of historical marine sea level pressure. J. Climate, 13, 2987-3002.

Kobayashi, T., and G. C. Johnson, 2007: Argo float pressure offset adjustment recommendations. Eighth Meeting of the Int. Argo Steering Team, Paris, France, IOC, 21-39. [Available online at http://prelude.ocean.washington.edu/dmqc3/pub/argo_float_ press_offset_adjustment.pdf.]

Leuliette, E. W., and L. Miller, 2009: Closing the sea level rise budget with altimetry, Argo, and GRACE. Geophys. Res. Lett., 36, L04608, doi:10.1029/2008GL036010.

Levitus, S., J. I. Antonov, T. P. Boyer, H. E. Garcia, and R. A. Locarnini, 2005: Linear trends of zonally averaged thermosteric, halosteric, and total steric sea level for individual ocean basins and the world ocean, (1955-1959)-(1994-1998). Geophys. Res. Lett., 32, L16601, doi:10.1029/2005GL023761.

,,,--- R. A. Locarnini, H. E. Garcia, and A. V. Mishonov, 2009: Global ocean heat content 1955-2008 in light of recently revealed instrumentation problems. Geophys. Res. Lett., 36, L07608, doi:10.1029/2008GL037155.

Lyman, J. M., J. K. Willis, and G. C. Johnson, 2006: Recent cooling of the upper ocean. Geophys. Res. Lett., 33, L18604, doi:10.1029/ 2006GL027033.

Murphy, D. M., S. Solomon, R. W. Portmann, K. H. Rosenlof, P. M. Forster, and T. Wong, 2009: An observationally based energy balance for the Earth since 1950. J. Geophys. Res., 114, D17107, doi:10.1029/2009jd012105.

Roemmich, D., and J. Gilson, 2009: The 2004-2008 mean and annual cycle of temperature, salinity, and steric height in the global ocean from the Argo Program. Prog. Oceanogr., 82, 81100, doi:10.1016/j.pocean.2009.03.004.

, and Coauthors, 1999: On the design and implementation of Argo: An initial plan for a global array of profiling floats. International CLIVAR Project Office Rep. 21, GODAE International Project Office Rep. 5., 32 pp. 
- and Coauthors, 2009: The Argo program: Observing the global oceans with profiling floats. Oceanography, 22 (2), 34-43.

Solomon, S., and Coauthors, 2007: Technical summary. Climate Change 2007: The Physical Science Basis, S. Solomon et al., Eds., Cambridge University Press, 19-91.

Uchida, H., and S. Imawaki, 2008: Estimation of the sea level trend south of Japan by combining satellite altimeter data with in-situ hydrographic data. J. Geophys. Res., 113, C09035, doi:10.1029/2008JC004796.

-, T. Kawano, and M. Fukasawa, 2008: In-situ calibration of moored CTDs used for monitoring abyssal water. J. Atmos. Oceanic Technol., 25, 1695-1702.
Wijffels, S. E., J. Willis, C. M. Domingues, P. Barker, N. J. White, A. Gronell, K. Ridgway, and J. A. Church, 2008: Changing expendable bathythermograph fall rates and their impact on estimates of thermosteric sea level rise. J. Climate, 21, 56575672.

Willis, J. K., J. M. Lyman, G. C. Johnson, and J. Gilson, 2007: Correction to "Recent cooling of the upper ocean". Geophys. Res. Lett., 34, L16601, doi:10.1029/2007GL030323.

— D. D. Chambers, and R. Nerem, 2008: Assessing the globally averaged sea level budget on seasonal to interannual timescales. J. Geophys. Res., 113, C06015, doi:10.1029/ 2007JC004517. 